\title{
Influences on amplitude estimation using the three- parameter sine fitting algorithm in the velocity mode of the Planck-Balance
}

\author{
Shan Lin ${ }^{1}$, Christian Rothleitner ${ }^{1}$, Norbert Rogge ${ }^{2}$, Thomas Fröhlich² \\ ${ }_{1}$ Physikalisch-Technische Bundesanstalt, Bundesallee 100, 38116 Braunschweig, Germany \\ ${ }^{2}$ Institute of Process Measurement and Sensor Technology, Technische Universität Ilmenau, 98684 IImenau, Germany
}

\begin{abstract}
The Planck-Balance is a table-top version of a Kibble balance. In contrast to many other Kibble balances, the coil in the Planck-Balance is moved sinusoidally and an AC rather than a DC signal is generated in velocity mode. The three-parameter sine fitting algorithm is applied to estimate the amplitudes of the induced voltage and the coil motion, which are used to determine the force factor $B /$ of the voice coil of the electromagnetic force compensation balance. However, the three-parameter sine fitting algorithm is not robust against some perturbations, e.g. additive Gaussian white noise, quantisation error, harmonic distortion, frequency error, and time jitter. These perturbations affect the accuracy of the amplitude estimation. Based on numerical simulations and correlation analyses, the effects of these perturbations are determined. By optimising the measurement and data processing approach, the bias and standard deviation of the estimated amplitude can be effectively reduced; thus, the accuracy of the force factor $B /$ in velocity mode can be improved.
\end{abstract}

Section: RESEARCH PAPER

Keywords: Planck-Balance; sine fitting algorithm; Monte Carlo simulation; Kibble balance

Citation: Shan Lin, Christian Rothleitner, Norbert Rogge, Thomas Fröhlich, Influences on amplitude estimation using the three-parameter sine fitting algorithm in the velocity mode of the Planck-Balance, Acta IMEKO, vol. 9, no. 3, article 7, September 2020, identifier: IMEKO-ACTA-09 (2020)-03-07

Editor: Jan Saliga, Technical University of Košice, Slovakia

Received January 17, 2020; In final form March 24, 2020; Published September 2020

Copyright: This is an open-access article distributed under the terms of the Creative Commons Attribution 3.0 License, which permits unrestricted use, distribution, and reproduction in any medium, provided the original author and source are credited.

Funding: This work was funded by 'Validierung des technologischen und gesellschaftlichen Innovationspotenzials - VIP+', a program of the German Federal Ministry of Education and Research (BMBF), which is managed by VDI/VDE Innovation + Technik GmbH.

Corresponding author: Shan Lin, e-mail: shan.lin@ptb.de

\section{INTRODUCTION}

After the redefinition of the kilogram, the Kibble balance is one possible approach to the calibration of mass standards in terms of the fixed value of the Planck constant with zero uncertainty [1]. The Planck-Balance (PB) is a table-top Kibble balance and is currently under development in a collaboration between Physikalisch-Technische Bundesanstalt (PTB) and Technische Universität Ilmenau (TUIL) [2].

The Kibble balance has two measuring modes: force mode and velocity mode. In the velocity mode of other Kibble balance experiments, the coil is usually moved at a constant velocity [3][5]. In contrast to these Kibble balances, the coil of the PB is sinusoidally moved through the magnetic ( $B$-)field in an oscillatory manner, thus inducing an AC voltage across the coil ends. This voltage is digitised by means of a high-precision digital multimeter (Keysight 3458A). If it is assumed that the coil motion and the induced voltage are perfectly sinusoidal, the force factor $B l$ ( $l$ denotes the coil wire length, $B$ the magnetic flux density) can be determined by the oscillation frequency, the amplitudes of the coil motion, and the induced voltage. In the PB setup, only the amplitudes need to be estimated because the oscillation frequency can be accurately measured with a frequency counter. The motion of the coil with respect to the magnet is measured with a high-precision (but commercial) laser interferometer, which provides the position data as a function of time. The measurements of voltage and position are synchronised by means of an external trigger source with a trigger frequency of $1 \mathrm{kHz}$.

The three-parameter sine fitting algorithm is applied to determine the amplitudes of the induced voltage and the coil motion. However, in practice, the sampled signal is not an ideal single-component sine wave due to some perturbations like, for example, additive Gaussian white noise, quantisation error, harmonic distortion, frequency error, and time jitter. The fitting 
algorithm is not robust against these perturbations and may result in a bias of the amplitude, which will directly affect the accuracy of Bl. In this article, the effects of aforementioned perturbations are analysed by numerical simulations. The bias and standard deviation of the estimated amplitude provided by the three-parameter sine fit are investigated by the theoretical equations and a Monte Carlo simulation.

\section{VELOCITY MODE OF THE PLANCK-BALANCE}

A Kibble balance establishes a link between the mass and the Planck constant. This is done by compensating the mechanical force $F_{m e c h}=m \cdot g$ with an electromagnetic force $F_{e m}=B$. $l \cdot I$. Here, $m$ denotes the mass, $g$ the local gravity, $B$ the magnetic flux density, $l$ the length of the coil in the magnetic field, and $I$ the electrical current that flows through the coil. The electromagnetic force is generated by a voice coil actuator. The electrical current can be measured to high precision via Ohm's law with an electrical resistor and a voltmeter, which can be traced back to quantum standards (the Quantum Hall resistor and Josephson voltage standard). $B$ and $l$, however, cannot be measured separately with high accuracy. This is why the so-called 'velocity mode' was introduced by Bryan Kibble (hence the name 'Kibble balance') [1]. Here, the coil is moved through the $B$-field that - according to Faraday's law - induces a voltage between the coil ends as $U_{\text {ind }}=B \cdot l \cdot v$, where $v$ denotes the coil velocity. In state-of-the-art Kibble balances, the motion has constant velocity. Hence, the induced voltage is DC. In the PB, the motion is harmonic. The induced voltage, therefore, is AC.

In the velocity mode of the PB, the coil oscillates through the $B$-field generated by the magnet. The coil position is measured by using an interferometer, while the induced voltage in the coil is measured by using a digital voltmeter synchronously. The measured coil motion is assumed to be an ideal singlecomponent sine wave with an amplitude $S$ and an initial phase $\varphi_{\mathrm{s}}$ :

$$
s(t)=S_{0}+S \sin \left(\omega t+\varphi_{\mathrm{s}}\right) .
$$

Here, $S_{0}$ is the DC offset and $\omega$ is the angular frequency, $\omega=2 \pi f_{\text {sig }}$, where $f_{\text {sig }}$ denotes the oscillation frequency.

The coil velocity can be obtained as the derivative of (1) with respect to time $t$ as

$$
v(t)=\omega S \cos \left(\omega t+\varphi_{\mathrm{s}}\right) .
$$

The induced voltage is also assumed to be a perfect sine wave with an amplitude $U$ and an initial phase $\varphi_{\mathrm{u}}$ :

$$
u(t)=U \cos \left(\omega t+\varphi_{\mathrm{u}}\right)
$$

Assuming that the force factor $B l$ is a constant during the whole range of the coil movement, $B /$ can be calculated to divide the amplitude of the induced voltage by the amplitude of the coil velocity as

$$
B l=\frac{U}{\omega S}=\frac{U}{2 \pi f_{\mathrm{sig}} S} .
$$

In the PB system, oscillation frequency $f_{\text {sig }}$ can be accurately measured by a frequency counter. Therefore, the accuracy of $B l$ depends mainly on the estimation of amplitudes $U$ and $S$.

\section{MEASUREMENT SETUP}

In the current setup of the PB (see Figure 1 [6]), which consists of several custom-made circuitries and a customised setup of commercially available devices, the controller is implemented on a Digital Signal Processor (DSP) system provided by dSPACE GmbH.

The control algorithm runs on a processor board of type DS1006, which is enhanced by an Analogue-to-Digital Converter (ADC) board of type DS2004 and a Digital-to-Analogue Converter (DAC) board of type DS2102. The available DACs and ADCs all have a 16-bit resolution.

The current PIDT $_{1}$-controller is implemented with a sampling frequency $f_{\mathrm{s}}$ of $10 \mathrm{kHz}$ and provides an output voltage $U_{\mathrm{drv}}$ in order to control the measured position sensor voltage $U_{\mathrm{P}}$ to a given set point value. The output voltage of the DSP is converted by an amplifier circuitry into a controlled current that is proportional to $U_{\text {drv }}$. When applied to the internal balance actuator, the output current can be utilised to excite the load carrier with a sinusoidal motion that is necessary in the velocity mode for the calibration of the force factor $\mathrm{Bl}$ of the external coil. After this calibration mode, the output current is connected via latching relays to the external coil and is then used to balance the system in force mode.

In addition to the control algorithm, several different signal generators are implemented on the DSP, which provide chirp signals for testing purposes and the sinusoidal modulation of the set point position in velocity mode. Since the internal clock of the DSP represents the time base for the excitation frequency, one of the DACs outputs a square wave that changes its voltage level at each sampling period of the DSP and therefore yields a frequency of $f_{\mathrm{s}} / 2$. The actual frequency of this signal is measured by a frequency counter of type Keysight 53220 A, which receives a reference frequency $f_{\text {ref }}=10 \mathrm{MHz}$ from a stable reference clock. The reference clock is an oven-stabilised quartz oscillator of type RSGGO10O provided by RF Suisse, which is disciplined by GPS signals.

The same reference frequency is supplied to a waveform generator of type Keysight $33521 \mathrm{~B}$ that provides a square wave signal with the frequency $f_{\text {trig. }}$. The square wave signal is used to trigger the measurement of the laser interferometer and the digital multimeters (Keysight 3458A) with a sampling frequency of $1 \mathrm{kHz}$. One multimeter measures the induced voltage in velocity mode, while the other one measures the necessary compensation current during force mode as a voltage drop $U_{\mathrm{r}}$

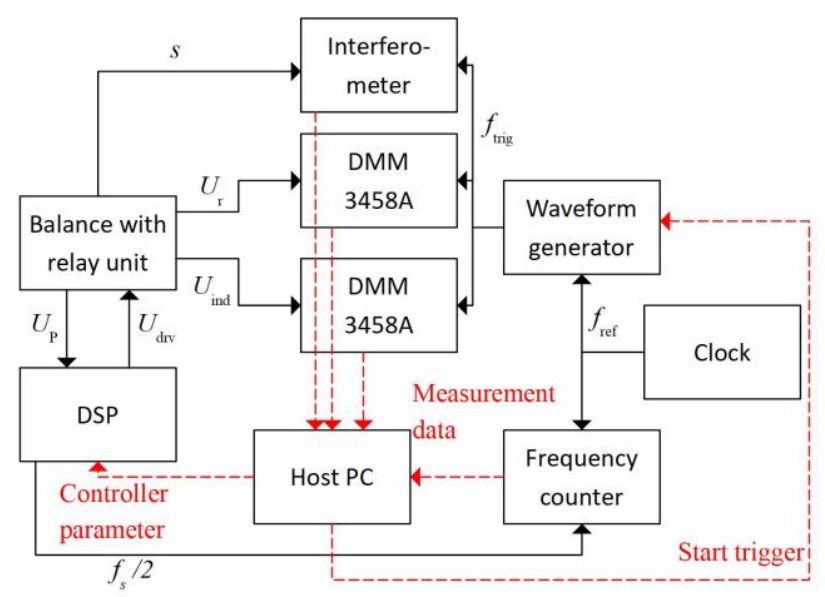

Figure 1. The signal processing setup of the PB [6] 
across a pre-calibrated reference shunt resistor that is connected in a series with the coil and the current amplifier output.

\section{THREE-PARAMETER SINE FIT}

A sinusoidal signal can be described as

$y(t)=Y_{0}+Y \sin (\omega t+\varphi)$,

where $Y$ is the amplitude, $\varphi$ the initial phase, and $Y_{0}$ a DC offset. Equivalently, the signal in (5) can be written as a linear combination of two shifted sine waves as

$$
y(t)=Y_{0}+A \sin (\omega t)+B \cos (\omega t)
$$

where $A$ and $B$ are the amplitudes of the in-phase and in-quadrature components, respectively. In (6), the signal frequency is known; thus, only three parameters $Y_{0}, A$, and $B$ need to be estimated.

When a set of $M$ (with $M>3$ ) samples $y_{1}, y_{2}, \ldots, y_{M}$ from a sine wave is sampled at the time instants $t_{1}, t_{2}, \ldots, t_{M}$, a linear least squares method can be used to determine the best sine wave parameters by minimising the sum of the squares of the following errors:

$$
\min _{A, B, Y_{0}} \sum_{i=1}^{M}\left(y_{i}-Y_{0}-A \sin \left(\omega t_{i}\right)-B \cos \left(\omega t_{i}\right)\right)^{2} \text {. }
$$

The estimated parameters of the sine wave can be calculated in a matrix form as:

$$
\left[\begin{array}{c}
A \\
B \\
Y_{0}
\end{array}\right]=\left(\mathbf{D}^{\mathrm{T}} \mathbf{D}\right)^{-1} \mathbf{D}^{\mathrm{T}}\left[\begin{array}{c}
y_{1} \\
y_{2} \\
\vdots \\
y_{M}
\end{array}\right],
$$

with

$$
\mathbf{D}=\left[\begin{array}{ccc}
\sin \left(\omega t_{1}\right) & \cos \left(\omega t_{1}\right) & 1 \\
\sin \left(\omega t_{2}\right) & \cos \left(\omega t_{2}\right) & 1 \\
\vdots & \vdots & \vdots \\
\sin \left(\omega t_{M}\right) & \cos \left(\omega t_{M}\right) & 1
\end{array}\right] .
$$

Amplitude $Y$ can be determined from $Y=\sqrt{A^{2}+B^{2}}$.

\section{INFLUENCES ON THE AMPLITUDE ESTIMATION}

The amplitudes of the induced voltage and displacement are provided by the sine fitting algorithm. An overview of the possible influences of the induced voltage on the amplitude estimation is given in Figure 2. Due to the possible error sources, the effects of these error sources on the amplitude estimation should be investigated, and the additional contributions to the uncertainty budget of the $B l$ in velocity mode must be considered. In this section, several error sources shown in Figure 2 are investigated by using the theoretical equation and/or numerical simulations. The values of most of the parameters used for the numerical experiments have been chosen according to real measurement data [7].

\subsection{Additive Gaussian white noise}

The amplitude estimation is affected by Additive Gaussian White Noise (AGWN), with zero mean and standard deviation $\sigma_{\mathrm{n}}$. The theoretical expressions have been derived in [8] and [9] as:

$$
\begin{aligned}
\varepsilon_{\mathrm{u}, \mathrm{N}} & \approx \frac{\sigma_{\mathrm{n}}^{2}}{M U_{\mathrm{ref}}^{2}} \text { and } \\
\sigma_{\mathrm{u}, \mathrm{N}} & =\sqrt{\frac{2}{M}} \sigma_{\mathrm{n}},
\end{aligned}
$$

where $\varepsilon_{\mathrm{u}, \mathrm{N}}$ and $\sigma_{\mathrm{u}, \mathrm{N}}$ are the systemic error and standard deviation of the estimated amplitude, respectively. $U_{\text {ref }}$ is the nominal i.e. unbiased voltage, and $M$ is the number of samples calculated from the product of sampling frequency $f_{\mathrm{s}}$ and sampling period $T$.

In order to validate the theoretical (9) and (10), the Monte Carlo Method (MCM) is used to evaluate the relative bias and standard deviation of the amplitude. Firstly, a single-component sine wave for the induced voltage is generated with the amplitude $U_{\text {ref }}=0.3051 \mathrm{~V}$, the initial phase $\varphi=-1.5876 \mathrm{rad}$, and the oscillation frequency $f_{\text {sig }}=4 \mathrm{~Hz}$. For the error comparison of amplitude, the value of $U_{\text {ref }}$ is taken as the reference value. A dataset $\left\{u_{i}\right\}_{i=1}^{10^{4}}$ is generated from the pure sine wave with the sampling frequency $f_{\mathrm{s}}=1 \mathrm{kHz}$ and the sampling period $T=10 \mathrm{~s}$.

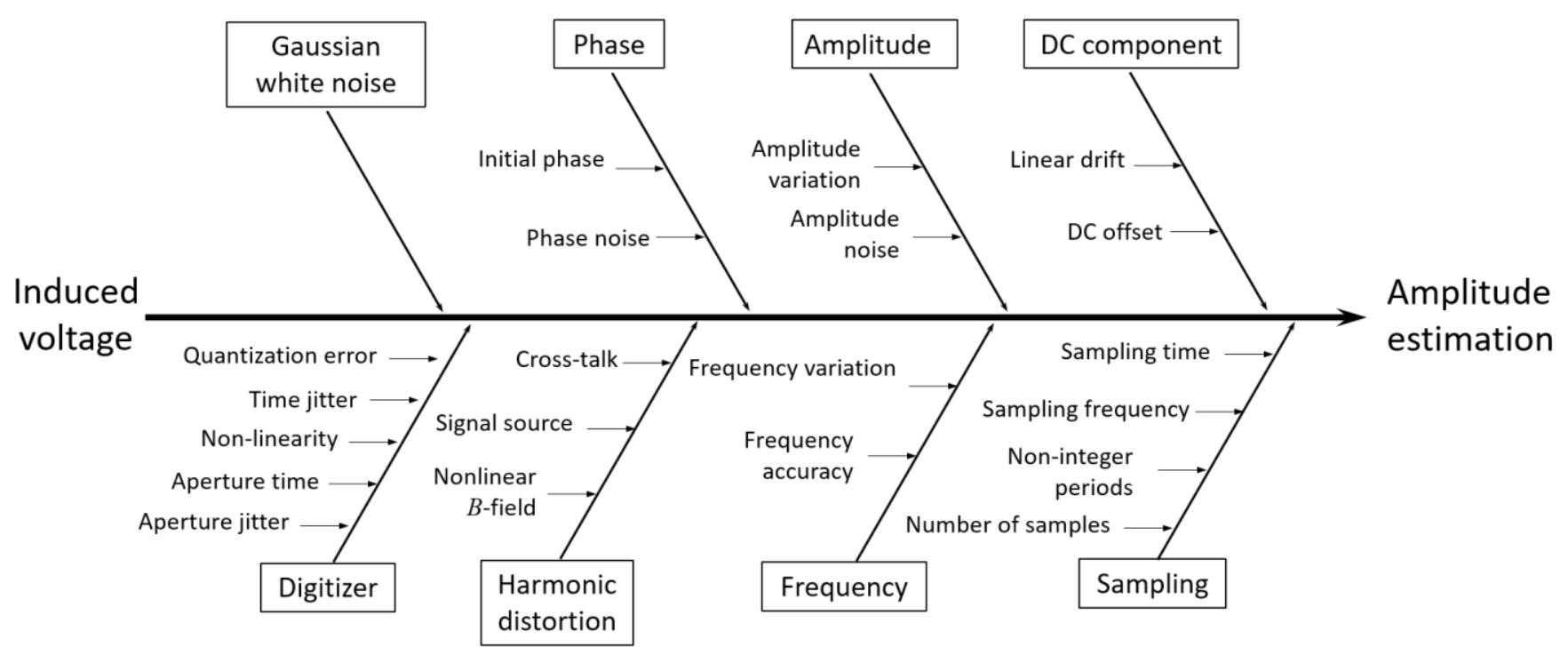

Figure 2. An Ishikawa diagram showing the influences of the possible error sources on the amplitude estimation of the induced voltage. 


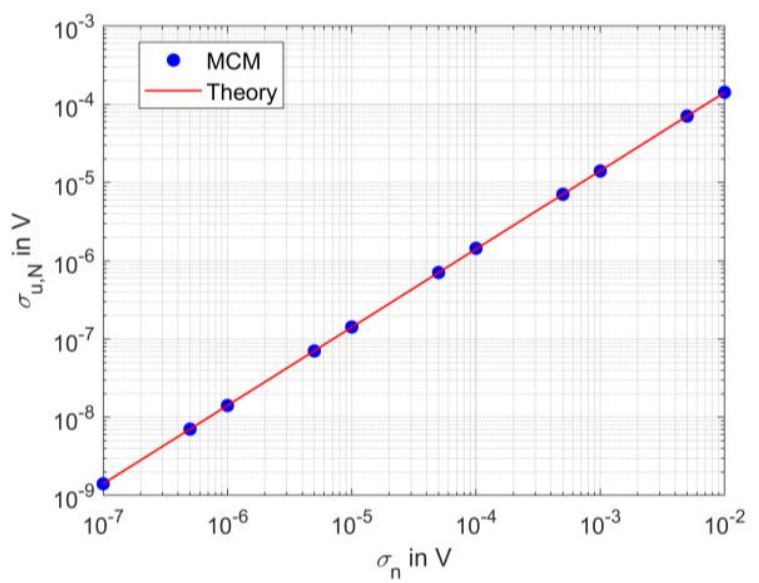

Figure 3. The standard deviation of the amplitude as a function of the noise standard deviation. The circles represent the simulated values obtained by the MCM. The solid line represents the theoretical values given by (10).

The generated dataset $\left\{u_{i}\right\}_{i=1}^{10^{4}}$ is taken as nominal points. The AGWN with different values of $\sigma_{\mathrm{n}}$ is superimposed on the nominal points. The three-parameter sine-fitting algorithm is used to estimate the amplitude $\widehat{U}$. The process is repeated $10^{4}$ times. Finally, the relative bias of the amplitude $\varepsilon_{\mathrm{u}, \mathrm{N}}$ is calculated by comparison of the mean value of $\widehat{U}$ with $U_{\text {ref }}$ as $\varepsilon_{\mathrm{u}, \mathrm{N}}=$ $\left|\widehat{U}-U_{\text {ref }}\right| / U_{\text {ref. }}$ The standard deviations associated with the estimated amplitude as a function of the noise level are presented in Figure 3. According to (10), the theoretical values are also calculated with $M=10^{4}$ samples and different values of $\sigma_{\mathrm{n}}$. The solid line in Figure 3 represents the theoretical results.

From (9) and (10), it can be deduced that the bias is negligible with respect to its standard deviation. Figure 3 shows good agreement between the theoretical and simulated results. It is shown that $\sigma_{\mathrm{u}, \mathrm{N}}$ increases with increasing $\sigma_{\mathrm{n}}$.

In the real measurement of $\mathrm{PB}$, the standard deviation of the residuals is in the order of $10^{-4} \mathrm{~V}$ after the extraction of the harmonics up to the fifth order. In such a case, the corresponding $\sigma_{\mathrm{u}, \mathrm{N}}$ is in the order of $10^{-6} \mathrm{~V}$. When the Type A evaluation is used to estimate the standard uncertainty, repeated measurements for the velocity mode can reduce the standard uncertainty of amplitudes. For the Type B evaluation, $\sigma_{\mathrm{u}, \mathrm{N}}$ can be reduced by either reducing $\sigma_{\mathrm{n}}$ or by increasing the number of samples $M$ for each single measurement i.e. the sampling frequency or the sampling period.

\subsection{Sampling strategy}

When measuring the induced voltage and the coil motion, the sampling frequency $f_{\mathrm{s}}$ and the sampling period $T$ need to be determined. The number of samples $M$ is equal to the product of $f_{\mathrm{s}}$ and $T$. As mentioned in Section 5.1, the bias of the estimated amplitude and associated standard deviation can be reduced by increasing $M$. The longer sampling period of measurement $T$ can increase the number of samples with a fixed sampling frequency $f_{\mathrm{s}}$.

In addition, more samples can also be obtained by increasing $f_{\mathrm{s}}$ within a fixed sampling period T. However, a higher sampling frequency $f_{\mathrm{s}}$ may result in a lower amplitude resolution of the digitiser with $q$ bits of resolution, which results in an increased quantisation error. The quantisation error is assumed to be uniformly distributed with the standard deviation $\sigma_{\mathrm{q}}[10]$ :

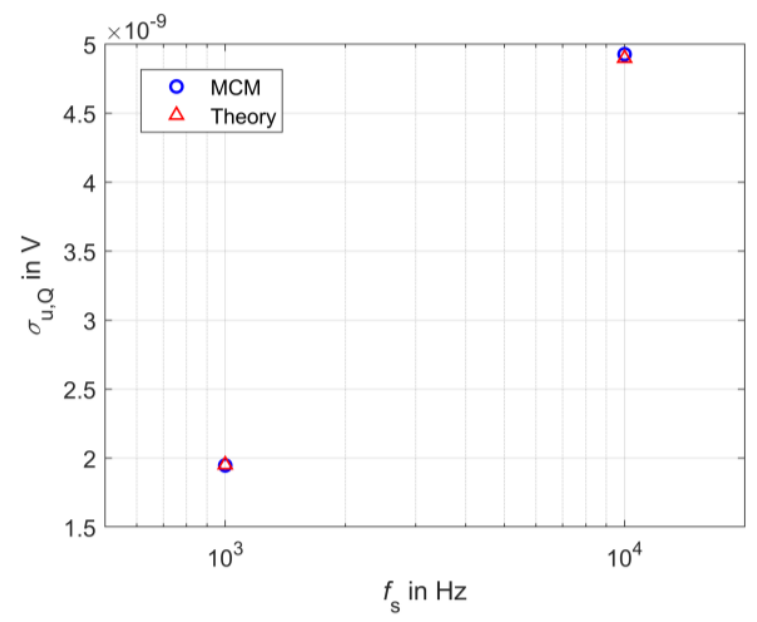

Figure 4. Standard deviation of the amplitude as a function of the sampling frequency. The circles represent the simulated values by the MCM. The triangles represent the theoretical values given by (12).

$$
\sigma_{\mathrm{q}}=\frac{1}{\sqrt{12}} \cdot \frac{U_{\mathrm{FSR}}}{2^{q}},
$$

where $U_{\mathrm{FSR}}$ is the full-scale range.

If only the quantisation noise is considered, the standard deviation of the estimated amplitude is [11]:

$$
\sigma_{\mathrm{u}, \mathrm{Q}}=\sqrt{\frac{2}{M}} \sigma_{\mathrm{q}} .
$$

Figure 4 gives an example of the influence of the quantisation noise on the amplitude estimation. The theoretical values with respect to sampling frequencies of $1 \mathrm{kHz}$ and $10 \mathrm{kHz}$ are represented by the blue circles, which are calculated by using (12). The digital multimeter Keysight 3458A is used to measure the induced voltage in a measurement range of $1 \mathrm{~V}$ i.e. $U_{\mathrm{FSR}}=1 \mathrm{~V}$. When the sampling frequency $f_{\mathrm{s}}=1 \mathrm{kHz}$, the corresponding resolution is 21 bits [12]. According to (11), $\sigma_{\mathrm{q}}$ is equal to $1.38 \times 10^{-7} \mathrm{~V}$. When the sampling period is $T=10 \mathrm{~s}$, the standard deviation of the voltage is $\sigma_{\mathrm{u}, \mathrm{Q}}=1.95 \times 10^{-9} \mathrm{~V}$. For a sampling frequency of $10 \mathrm{kHz}$, the resolution is $q=18 \mathrm{bits}$, and $\sigma_{\mathrm{q}}=1.10 \times 10^{-6} \mathrm{~V}$. Consequently, $\sigma_{\mathrm{u}, \mathrm{Q}}$ is equal to $4.92 \times 10^{-9} \mathrm{~V}$.

Next, the MCM is implemented to evaluate the standard deviation of the amplitude. Two datasets of nominal points are generated i.e. $\left\{u_{i}\right\}_{i=1}^{10^{4}}$ for $f_{\mathrm{s}}=1 \mathrm{kHz}$ and $\left\{u_{i}\right\}_{i=1}^{10^{5}}$ for $f_{\mathrm{s}}=10 \mathrm{kHz}$. The quantisation noise is generated and superimposed on the nominal points. The process is repeated $10^{4}$ times. The standard deviations of the estimated amplitude are represented by red triangles in Figure 4. The results show that those theoretically determined values have been validated by the MCM. When the sampling frequency $f_{\mathrm{s}}$ increases by a factor of ten from $1 \mathrm{kHz}$ to $10 \mathrm{kHz}$, the corresponding standard deviation of amplitude increases by a factor of about 2.5 (see Figure 4). However, $\sigma_{\mathrm{u}, \mathrm{Q}}$ is in the order of $10^{-9}$, which is much lower than the noise level. Therefore, the quantisation error is negligible in this case.

If $f_{\mathrm{s}}=10 \mathrm{kHz}$ is used instead of $1 \mathrm{kHz}$ and the noise standard deviation of $\sigma_{\mathrm{n}}=10^{-4} \mathrm{~V}$, the corresponding $\sigma_{\mathrm{u}, \mathrm{N}}$ is reduced by $\sqrt{10}$ times according to (10). Therefore, $f_{\mathrm{s}}=10 \mathrm{kHz}$ can be adopted to reduce the uncertainty of amplitude estimation. 


\subsection{Harmonic distortion}

The oscillation frequency $f_{\text {sig, }}$, the sampling frequency $f_{\text {s }}$, and the number of samples $M$ satisfy the relation [13]

$$
\frac{f_{\text {sig }}}{f_{\mathrm{s}}}=\frac{J+\delta}{M},
$$

where $J$ and $\delta$ are, respectively, the integer and the fractional parts of the number of sine wave cycles.

When $\delta=0$, it is called coherent sampling, and (9) and (10) are only valid in this case. However, when non-coherent sampling $(\delta \neq 0)$ occurs, the three-parameter sine fit provides a biased amplitude due to harmonic distortion [14]. Multiharmonic sine fitting or the extraction of integer periods of the sine wave can be used to reduce the bias of the amplitude provided by the three-parameter sine fit.

In practice, there are higher-order harmonics in the measurements of the induced voltage and the displacement. The magnitudes of higher-order amplitudes are shown in Figure 5 by using a Fast Fourier Transform (FFT) - obtained from a real measurement of the induced voltage. The oscillation frequency of the measurement data in Figure 5 is about $4 \mathrm{~Hz}$. The amplitudes of the second- up to the fifth-order harmonics are estimated by a factor of about $10^{3}, 10^{4}, 10^{5}$, and $10^{5}$ smaller than that of the first harmonic, respectively. The total harmonic distortion is $0.15 \%$.

A numerical simulation is implemented to investigate the influence of the harmonic distortion on the amplitude estimation. Figure 6 shows the simulation results [15]. The signal of the induced voltage is simulated by using a fundamental sine wave and simply superimposed by higher order harmonics up to the fifth order. The adopted amplitude for each harmonic is acquired from the real measurement data, which is the same amplitude as that which is shown in Figure 5. The oscillation frequency $f_{\text {sig }}$ is changed from $3.82 \mathrm{~Hz}$ to $4.02 \mathrm{~Hz}$ with the same sampling frequency of $f_{\mathrm{s}}=1 \mathrm{kHz}$ and the sampling period of $T=10 \mathrm{~s}$. When the oscillation frequency is $f_{\text {sig }}=3.9 \mathrm{~Hz}$ or $4 \mathrm{~Hz}$, the number of sine wave cycles is integer i.e. we have coherent sampling. Figure 6 shows that sine-fitting algorithms are robust against harmonics distortion when coherent sampling is applied. In such cases, the bias of the amplitude can be negligible. However, for the non-coherent sampling, the relative bias of the amplitude provided by the three-parameter sine fit $(N=1)$ is in the order of $10^{-6}$. Compared to the target lowest relative standard

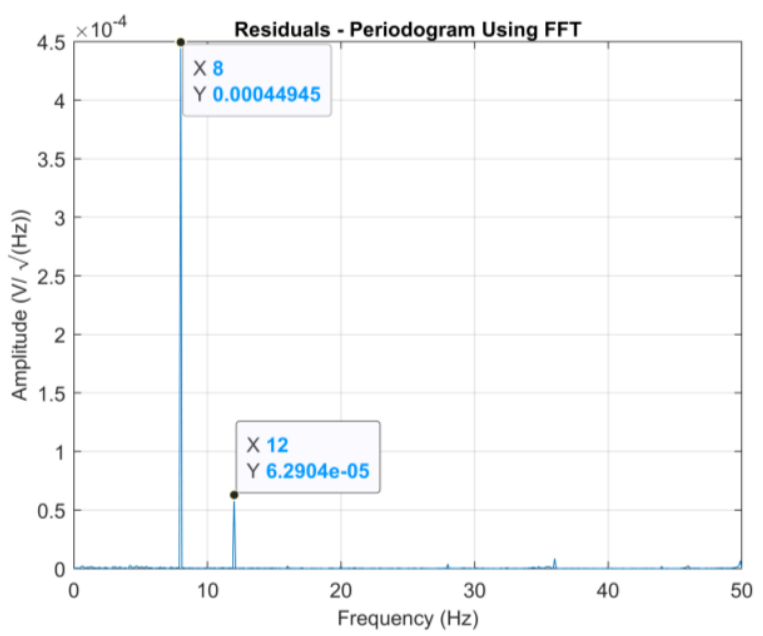

Figure 5. A periodogram using FFT for the residuals obtained by using the three-parameter sine fit. uncertainty for the PB $\left(8.4 \times 10^{-8}\right.$ for $\left.1 \mathrm{~kg}\right)$, the bias of the amplitude is relatively high. When the multiharmonic sine fit is applied, the higher the number of the included harmonics, the lower the bias of the estimated amplitude. The relative bias of the amplitude can greatly be reduced (to below $10^{-10}$ ) for the noncoherent sampling if the number of samples is reduced to obtain an integer number of periods (the green line in Figure 6). In such cases, the bias of the estimated amplitude becomes negligible.

Higher-order harmonics are present in the real measurement of the induced voltage as well as in the coil motion, which may be caused by the current source, nonlinear $B$-field, and so on. Moreover, in practice, coherent sampling cannot be perfectly achieved due to the accuracy of the generated frequency from the waveform generator - the time jitter of the sampling. Therefore, the extraction of the integer periods of the sine wave or the multiharmonic sine fitting included higher-order harmonics that could be adopted to estimate the amplitudes of induced voltage and coil motion.

\subsection{Frequency error}

In the linear sine-fitting algorithm, the oscillation frequency $f_{\text {sig }}$ is assumed to be known, and it is taken as an input parameter to the fitting model. If there is an error $\delta f_{\text {sig }}$ in the input frequency, an additional contribution will be included in the fitting model, which is described by a linearly damped oscillation [16].

A numerical simulation has been implemented in order to investigate the effect produced by a frequency error $\delta f_{\text {sig. }}$. The dataset $\left\{u_{i}\right\}_{i=1}^{10^{4}}$ is generated with $f_{\text {sig }}=10 \mathrm{~Hz}, f_{\mathrm{s}}=1 \mathrm{kHz}$, and $T=10 \mathrm{~s}$. A frequency error $\delta f_{\text {sig }}$ is added to $f_{\text {sig }}$, and the obtained frequency $\left(\delta f_{\text {sig }}+f_{\text {sig }}\right)$ is taken as the known frequency of the three-parameter sine fit to evaluate the amplitude. Finally, the relative bias of the amplitude $\varepsilon_{\mathrm{u}, \mathrm{F}}$ is calculated and shown in Figure 7.

It can be seen from Figure 6 that in order to keep this bias below $10^{-8}$, the frequency has to be known with the same relative uncertainty. In the measurement setup, the frequency counter of type Keysight $53220 \mathrm{~A}$ can measure the actual frequency accurately $\left(<10^{-8}\right)$, which can be used as the input frequency of the model and can achieve the desired accuracies for the PB (about $10^{-8}$ ). Experiments using other algorithms (e.g. the four-

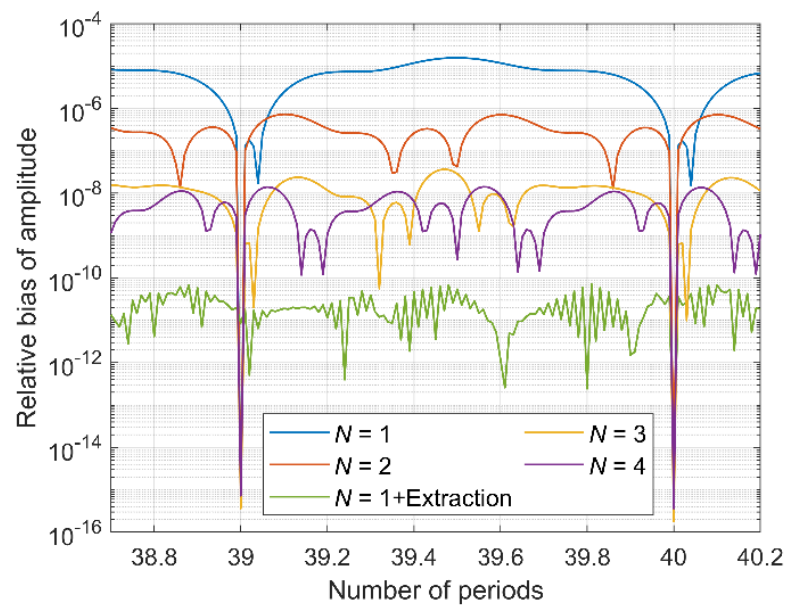

Figure 6 . The relative bias of the amplitude as a function of the number of periods [15]. The amplitudes are estimated by using multiharmonic sine fitting included from first- up to fourth-order harmonics and the extraction of the integer periods of the sine wave. 


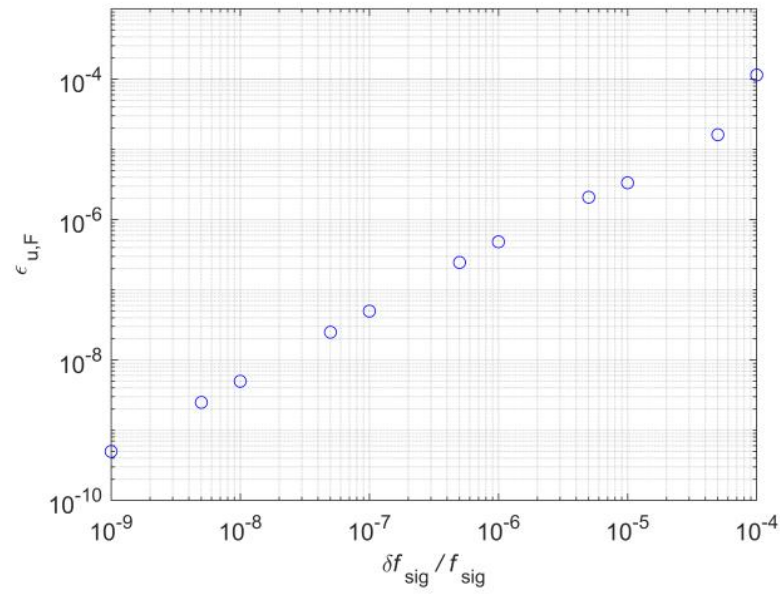

Figure 7. The relative bias of the amplitude as a function of the relative error of the oscillation frequency.

parameter sine fit) showed that those algorithms can also achieve frequency estimations with errors in the order of $10^{-8}[13]$.

\subsection{Time jitter}

When implementing the sine fitting algorithm in (7), it is assumed that the sampling instants $t_{1}, t_{2}, \ldots, t_{M}$ have no influence due to the time jitter. However, in practice, the time jitter is an unpredictable timing noise, and this effect leads to the bias of estimated sine wave amplitude. The relative bias of the amplitude is [17]

$$
\varepsilon_{\mathrm{u}, \mathrm{J}}=\left(\frac{1}{2}-\frac{1}{M}\right)\left(e^{-\sigma_{\theta}^{2}}-1\right)
$$

where $\sigma_{\theta}=2 \pi f_{\text {sig }} \sigma_{\mathrm{t}}$, and $\sigma_{\mathrm{t}}$ is the standard deviation of the time jitter.

Moreover, the time jitter also leads to amplitude noise of the estimated signal. The corresponding noise standard deviation of the amplitude is calculated as [18]:

$$
\sigma_{\mathrm{j}}=2 \pi A_{\mathrm{RMS}} f_{\mathrm{sig}} \sigma_{\mathrm{t}},
$$

where $A_{\mathrm{RMS}}$ is the Maximum Root Mean Square (RMS) amplitude of the effective value of an AC signal.

As the induced voltage is an AC measurement, the maximum RMS amplitude $A_{\text {RMS }}$ is equal to $U_{\text {ref }} / \sqrt{2}$. If only the time jitter is considered, the standard deviation of the estimated amplitude is

$$
\sigma_{\mathrm{u}, \mathrm{J}}=\sqrt{\frac{2}{M}} \pi U_{\mathrm{ref}} f_{\mathrm{sig}} \sigma_{\mathrm{t}} .
$$

The time jitter of a Keysight 3458A DMM is 5 ns and has a rectangular distribution [19]. Thus, the standard deviation of the time jitter becomes $\sigma_{\mathrm{t}}=2.89 \mathrm{~ns}$. When $f_{\text {sig }}=4 \mathrm{~Hz}$ and $M=10^{4}$, the relative bias and relative standard deviation of the amplitude is in the order of $10^{-15}$ and $10^{-10}$, respectively. In such a case, the influences of the time jitter are negligible.

According to (14) and (16), the influences of the time jitter will be stronger for a sampled signal with a higher oscillation frequency and a lower number of samples. In the current measurement of the $\mathrm{PB}$, the number of the sample points is not below 5000, and the oscillation frequency is lower than $15 \mathrm{~Hz}$. If $f_{\text {sig }}=15 \mathrm{~Hz}$ and $M=5000$ are selected to estimate the relative bias and relative standard deviation, in the worst-case scenario, the values are equal to $3.7 \times 10^{-14}$ and $2.7 \times 10^{-9}$, respectively. Therefore, the time jitter is negligible in the current measurement

\section{CONCLUSIONS}

Perturbations in the signal can result in a bias of the amplitude estimation when using the three-parameter sine-fitting algorithm. The effects of AGWN, quantisation error, harmonic distortion, frequency error, and time jitter have been investigated by numerical simulations in this article. The bias and the associated standard deviation due to AGWN depend on the noise level and the number of samples. In order to improve the accuracy of the amplitude estimation, a sampling frequency $f_{\mathrm{s}}=10 \mathrm{kHz}$ can be used instead of $1 \mathrm{kHz}$ without notable influence of the increased quantisation noise. The three-parameter sine fit is not robust against harmonic distortion with non-coherent sampling. In order to reduce the bias of the estimated amplitude, the extraction of the integer periods of the sine wave or the multiharmonic sine fitting included higher-order harmonics could be adopted. Moreover, the simulation results indicate that the frequency error of the fitting mode can also cause a biased amplitude. However, the use of a frequency counter or some effective algorithms can keep this influence negligible. The effects of time jitter can also be neglected in this case. In total, it can be concluded that the three-parameter sine-fitting algorithm satisfies the requirement of the PB.

As a next step, work will be carried out in order to include other important effects, e.g. a nonlinear B-field. All the investigated error sources will be taken into account in order to evaluate the uncertainty of the force factor $B l$ in the velocity mode. The aim is to create a good model for the implementation in a digital twin of the Planck-Balance i.e. a Monte Carlo-based uncertainty estimation to the PB [2].

\section{ACKNOWLEDGEMENT}

The research of this project is funded via the program 'Validierung des technologischen und gesellschaftlichen Innovationspotenzials - VIP+', a program of the German Federal Ministry of Education and Research (BMBF), which is managed by VDI/VDE Innovation + Technik GmbH. The authors are grateful to the members of the Planck-Balance team (PTB + TUIL) for our fruitful discussions.

This article is an extended version of a contribution to the $23^{\text {rd }}$ IMEKO TC4 International Symposium in Xi'an, China [20].

\section{REFERENCES}

[1] I. A. Robinson, S. Schlamminger, The watt or Kibble balance: a technique for implementing the new SI definition of the unit of mass, Metrologia 53(5) (2016) pp. A46-A74. Online [Accessed 24 September 2020]

DOI: https://doi.org/10.1088/0026-1394/53/5/A46

[2] C. Rothleitner, J. Schleichert, N. Rogge, L. Günther, S. Vasilyan, F. Hilbrunner, D. Knopf, T. Fröhlich, F. Härtig, The PlanckBalance - using a fixed value of the Planck constant to calibrate E1/E2-weights, Meas. Sci. Technol. 29(7) (2018) pp. 074003. DOI: https://doi.org/10.1088/1361-6501/aabc9e

[3] I. A. Robinson, Towards the redefinition of the kilogram: a measurement of the Planck constant using the NPL Mark II watt balance, Metrologia 49(1) (2012) pp. 113-156. DOI: https://doi.org/10.1088/0026-1394/49/1/016

[4] H. Fang, A. Kiss, E. de Mirandés, J. Lan, L. Robertsson, S. Solve, A. Picard, M. Stock, Status of the BIPM watt balance, IEEE Trans. Instrum. Meas., 62(6) (2013) pp. 1491-1498. DOI: https://doi.org/10.1109/TIM.2012.2225930 
[5] D. Haddad, F. Seifert, L. S. Chao, S. Li, D. B. Newell, J. R. Pratt, C. Williams, S. Schlamminger, Invited article: A precise instrument to determine the Planck constant, and the future kilogram, Rev. Sci. Instrum. 87(6) (2016) pp. 061301. Online [Accessed 24 September 2020] DOI: https://doi.org/10.1063/1.4953825

[6] N. Rogge, S. Lin, C. Rothleitner, S. Vasilyan, Excitation frequency dependent deviations during the "velocity mode" of $\mathrm{Bl}$ measurements in the Planck-Balance, Proc. of $23^{\text {rd }}$ IMEKO TC4 International Symposium, 17-20 September 2019, Xi'an, China. Online [Accessed 24 September 2020]

URL: https://www.imeko.org/publications/tc4-2019/IMEKOTC4-2019-044.pdf

[7] C. Rothleitner, J. Schleichert, S. Vasilyan, N. Rogge, L. Günther, F. Hilbrunner, I. Rahneberg, D. Knopf, T. Fröhlich, F. Härtig, First results using the Planck-Balance, Proc. of the 2018 Conference on Precision Electromagnetic Measurements (CPEM 2018), 8-13 July 2018, Paris, France.

DOI: https://doi.org/10.1109/CPEM.2018.8500904

[8] F. Corrêa Alegria, Bias of amplitude estimation using threeparameter sine fitting, Measurement 42(5) (2009) pp. 748-756. DOI: https://doi.org/10.1016/i.measurement.2008.12.006

[9] M. Martino, R. Losito, A. Masi, Analytical metrological characterization of the three-parameter sine fit algorithm, ISA Transactions 51(2) (2012) pp. 262-270.

DOI: https://doi.org/10.1016/j.isatra.2011.10.003

[10] IEEE Std. 1241, IEEE Standard for Terminology and Test Methods for Analog-to-Digital Converters, IEEE, 2000.

[11] F. Corrêa Alegria, A. Cruz Serra, Uncertainty of the estimates of sine wave fitting of digital data in the presence of additive noise, Proc. of the IEEE Instrumentation and Measurement Technology Conference, 24-27 April 2006, Sorrento, Italy, pp. 1643-1647. DOI: https://doi.org/10.1109/IMTC.2006.328187

[12] Keysight Technologies, Keysight 3458A Multimeter User's Guide, 2017.
[13] D. Petri, D. Belega, D. Dallet, Dynamic testing of analog-to-digital converters by means of the sine-fitting algorithms, in: Design, Modeling and Testing of Data Converters. P. Carbone, S. Kiaei, F. Xu (editors). Springer, Berlin, 2014, ISBN 978-3-642-39654-0, pp. 309-340.

[14] J. P. Deyst, T. M. Sounders, O. M. Solomon, Bounds on leastsquares four-parameter sine-fit errors due to harmonic distortion and noise, IEEE Trans. Instrum. Meas. 44(3) (1995) pp. 637-642. DOI: https://doi.org/10.1109/19.387298

[15] S. Lin, C. Rothleitner, N. Rogge, Investigations on the sine fitting algorithm in the Planck-Balance, Proc. of $20^{\text {th }}$ GMA/ITGFachtagung Sensoren und Messsysteme 2019, 25-26 June 2019, Nürnberg, Germany, pp. 547-553. Online [Accessed 24 September 2020]

DOI: https://doi.org/10.5162/sensoren2019/6.4.4

[16] A. Baccigalupi, G. d'Alessandro, M. D'Arco, R. S. L. Schiano, Least square procedures to improve the result of the threeparameter sine-fitting algorithm, Acta IMEKO 4(2) (2015) pp. 100-106. Online [Accessed 24 September 2020]

DOI: https://doi.org/10.21014/acta imeko.v4i2.173

[17] F. Corrêa Alegria, A. Cruz Serra, Gaussian jitter-induced bias of sine wave amplitude estimation using three-parameter sine fitting, IEEE Trans. Instrum. Meas. 59(9) (2010) pp. 2328-2333. DOI: https://doi.org/10.1109/TIM.2009.2034576

[18] R. Lapuh, Sampling with 3458A: Understanding, Programming, Sampling and Signal Processing, Left Right d.o.o., Ljubljana, Slovenia, 2018, ISBN 978-961-94476-0-4.

[19] W. G. Kuerten Ihlenfeld, Maintenance and traceability of AC voltage by synchronous digital synthesis and sampling, PTBBericht PTB-E-75, 2001, ISBN 3-89701-747-4.

[20] S. Lin, C. Rothleitner, N. Rogge, Amplitude estimation using three-parameter sine fitting algorithm in the Planck-Balance, Proc. of 23rd IMEKO TC4 International Symposium, 17-20 September 2019, Xi'an, China.

URL: https://www.imeko.org/publications/tc4-2019/IMEKOTC4-2019-019.pdf 